\title{
Bogomolny equations for vortices in the noncommutative torus
}

\author{
Peter Forgács \\ Laboratoire de Mathématiques et Physique Théorique, CNRS/UMR 6083 \\ Université de Tours \\ Parc de Grandmont, 37200 Tours, France \\ E-mail: forgacs@rmki.kfki.hu
}

\section{Gustavo S. Lozano*}

Departamento de Física, FCEyN, Universidad de Buenos Aires

Pab. 1, Ciudad Universitaria, Buenos Aires, Argentina

E-mail: lozano@df.uba.ar

\section{Enrique F. Moreno*}

Departamento de Fúsica, Facultad de Ciencias Exactas

Universidad Nacional de La Plata

C.C. 67, 1900 La Plata, Argentina, and

Department of Physics, West Virginia University

Morgantown, West Virginia 26506-6315, U.S.A.

E-mail: enrique.moreno@mail.wvu.edu

\section{Fidel A. Schaposnik ${ }^{\dagger}$}

Departamento de Física, Facultad de Ciencias Exactas

Universidad Nacional de La Plata

C.C. 67, 1900 La Plata, Argentina

E-mail: fidel@fisica.unlp.edu.ar

ABSTRACT: We derive Bogomolny-type equations for the abelian Higgs model defined on the noncommutative torus and discuss its vortex like solutions. To this end, we carefully analyze how periodic boundary conditions have to be handled in noncommutative space and discuss how vortex solutions are constructed. We also consider the extension to an $\mathrm{U}(2) \times \mathrm{U}(1)$ model, a simplified prototype of the noncommutative standard model.

Keywords: Solitons Monopoles and Instantons, Non-Commutative Geometry.

*Associated with CONICET.

${ }^{\dagger}$ Associated with CICBA. 


\section{Contents}

1. Introduction 1

2. Gauge and matter fields on the noncommutative torus 2

$\begin{array}{lll}\text { 3. The Maxwell-Higgs model } & 7\end{array}$

4. Non abelian extension and discussion $\quad 10$

A. A useful result $\quad 12$

B. Cyclic property of the integral $\quad 14$

\section{Introduction}

Construction of noncommutative solitons and instantons has been a field of intense activity after the revival of field theories in noncommutative space, in connection with string theory and brane dynamics (see for example [1] for references on this issue). Not only the noncommutative counterparts of vortices, monopoles and other localized solutions in ordinary space were constructed but regular stable solutions which become singular in the commutative limit were also discovered (see for example [2] for a complete list of references). Concerning static classical solutions of the abelian Higgs model in the noncommutative plane, both BPS and non BPS vortices have been constructed and its moduli space studied in detail [3].

In the present work we consider vortex solutions in the abelian Higgs model defined on the noncommutative torus and then extend the analysis to the case of a $U(2) \times U(1)$ symmetry. This is motivated by the fact that, in commutative space, one can find stable solutions that correspond to periodic arrays of vortices in theories with gauge field coupled to Higgs scalars. Moreover, the analysis of such kind of arrays is equivalent to the study of models defined on the torus. This fact has been exploited in the search of vortex solutions in the Salam-Weinberg model where the only stable solutions correspond to such type of arrays [4]. Hence our results can be seen as a first step along this line in its noncommutative version.

Despite the fact that the 2 dimensional torus is one of the simplest examples of noncommutative space, no discussion of the BPS equations and their solution for the MaxwellHiggs model has been carried out. In this respect, our work fills in this gap and also opens the possibility of studying non-abelian extensions related to the noncommutative version of the Salam-Weinberg theory. Bogomolny equations for the abelian Higgs model on a two dimensional torus have been first considered by Shah and Manton [5]. More recently, Gonzalez Arroyo and Ramos [6] have analyzed them in detail and presented a high precision approximation scheme. 
The paper is organized as follows: we introduce in section 2 the noncommutative torus $\mathcal{T}$ (and noncommutative parameter $\theta$ ) and discuss periodicity conditions for gauge and matter fields. We show that consistency of gauge transformations and periodicity conditions naturally leads to the introduction of a scaled torus $\overline{\mathcal{T}}$ and a $\theta$-depending scaled gauge charge. Then, in section 3 we discuss the dynamics of the Maxwell-Higgs model showing that the role of the scaled torus becomes crucial in the definition of gauge invariant expressions for the energy and magnetic flux as well as for the obtention of covariant BPS equations. We present a particular solution to these equations and we also discuss the strategy to obtain general vortex like solutions, analogous to that leading to numerical solutions in the commutative torus [6]. Finally, in section 4 we extend the discussion to the case of a $\mathrm{U}(2) \times \mathrm{U}(1)$ lagrangian for which we also write the BPS equations and indicate how one should look for their solution. We leave for an appendix the derivation of some results needed to implement periodic boundary conditions on the noncommutative torus.

\section{Gauge and matter fields on the noncommutative torus}

Let us consider noncommutative $2+1$ dimensional space-time with coordinates satisfying

$$
[x, y]=i \theta, \quad[x, t]=[y, t]=0 .
$$

Our model will be defined on a spatial torus $\mathcal{T}$ with periods $\left(L_{1}, L_{2}\right)$.

We shall be interested in a U(1) gauge theory with Higgs scalars $\phi$ in the fundamental representation coupled to gauge fields $A_{i}$. The fields transform under the $\mathrm{U}(1)$ gauge group according to

$$
\begin{aligned}
A_{i} \rightarrow A^{(V)} & =V^{-1} A_{i} V+\frac{i}{g} V^{-1} \partial_{i} V \\
\Phi \rightarrow \Phi^{(V)} & =V^{-1} \Phi
\end{aligned}
$$

As in ordinary space, a scalar field on the noncommutative torus can be defined as a function $\phi(x, y)$ which is periodic up to gauge transformations. That is,

$$
\begin{aligned}
& \phi\left(x+L_{1}, y\right)=U_{1}(x, y) \phi(x, y)=\phi^{U_{1}^{-1}}(x, y) \\
& \phi\left(x, y+L_{2}\right)=U_{2}(x, y) \phi(x, y)=\phi^{U_{2}^{-1}}(x, y)
\end{aligned}
$$

where $U_{1}$ and $U_{2}$ are $\mathrm{U}(1)$ gauge transformations. Concerning gauge fields, boundary conditions are

$$
\begin{aligned}
& A_{i}\left(x+L_{1}, y\right)=A_{i}^{\left(U_{1}^{-1}\right)}(x, y) \\
& A_{i}\left(x, y+L_{2}\right)=A_{i}^{\left(U_{2}^{-1}\right)}(x, y)
\end{aligned}
$$

Consistency of the precedent equations implies

$$
U_{2}\left(x+L_{1}, y\right) U_{1}(x, y)=U_{1}\left(x, y+L_{2}\right) U_{2}(x, y) .
$$

Note that eq. (2.7) coincides with the well-known consistency condition for the commutative torus (See for example [7] and references therein). 
A particular solution to eq. (2.7) is

$$
U_{1}(x, y)=e^{i \pi \omega L_{1} y}, \quad U_{2}(x, y)=e^{-i \pi \omega L_{2} x},
$$

where

$$
\omega=\frac{1}{\theta \pi}\left(1-\sqrt{1+\frac{2 \pi \theta k}{L_{1} L_{2}}}\right), \quad k \in \mathbb{Z} .
$$

It should be noted that in the $\theta \rightarrow 0$ limit, solution (2.8)-(2.9) goes smoothly to the solution on the commutative torus. One can make easily contact between this result and the discussion in $[8]$ on pure $\mathrm{U}(p)$ Yang-Mills theory on the noncommutative torus (in the particular $p=1$ and zero 't Hooft twist case).

Since $U_{1}$ and $U_{2}$ are translation generators, then for any arbitrary function $f(x, y)$ it holds that

$$
\begin{gathered}
U_{1}(x, y) f(x, y) U_{1}^{-1}(x, y)=f\left(x+\pi \omega L_{1} \theta, y\right) \\
U_{2}(x, y) f(x, y) U_{2}^{-1}(x, y)=f\left(x, y+\pi \omega L_{2} \theta\right) .
\end{gathered}
$$

Periodicity conditions (2.4) and the gauge transformation laws imply the following transformation laws for the transition functions under gauge transformations

$$
\begin{aligned}
& U_{1}(x, y) \rightarrow U_{1}^{\prime}(x, y)=V\left(x+L_{1}, y\right) U_{1}(x, y) V^{-1}(x, y) \\
& U_{2}(x, y) \rightarrow U_{2}^{\prime}(x, y)=V\left(x, y+L_{2}\right) U_{2}(x, y) V^{-1}(x, y) .
\end{aligned}
$$

Now, using property (2.10) we have

$$
\begin{aligned}
& U_{1}^{\prime}(x, y)=V\left(x+L_{1}, y\right) V^{-1}\left(x+\pi \omega L_{1} \theta, y\right) U_{1}(x, y) \\
& U_{2}^{\prime}(x, y)=V\left(x, y+L_{2}\right) V^{-1}\left(x, y+\pi \omega L_{2} \theta\right) U_{2}(x, y) .
\end{aligned}
$$

Then, if the gauge transformation functions are periodic with periods

$$
\tilde{L}_{i}=s L_{i}, \quad s=(1-\pi \omega \theta)=\sqrt{1+\frac{2 \pi \theta k}{L_{1} L_{2}}} \quad i=1,2
$$

the transition functions are invariant. Thus, we will restrict ourselves to gauge transformations satisfying this property. From now on, we shall call $\tilde{\mathcal{T}}$ the scaled torus with periods $\left(\tilde{L}_{1}, \tilde{L}_{2}\right)$.

The boundary conditions (2.6) together with our choice of transitions functions (2.8) imply for the gauge field the following equations

$$
\begin{aligned}
& A_{1}\left(x+L_{1}(1-\pi \omega \theta), y\right)=A_{1}(x, y) \\
& A_{1}\left(x, y+L_{2}(1-\pi \omega \theta)\right)=A_{1}(x, y)-\frac{1}{g} \pi \omega L_{2} \\
& A_{2}\left(x+L_{1}(1-\pi \omega \theta), y\right)=A_{2}(x, y)+\frac{1}{g} \pi \omega L_{1} \\
& A_{2}\left(x, y+L_{2}(1-\pi \omega \theta)\right)=A_{2}(x, y)
\end{aligned}
$$


which have as a general solution,

$$
A_{i}(x, y)=\tilde{A}_{i}(x, y)+a_{i}(x, y) .
$$

where $\tilde{A}_{i}$ is a periodic function in the scaled torus $\tilde{\mathcal{T}}$ and $a_{i}$ is defined as

$$
a_{i}=f \varepsilon_{i j} x^{j}
$$

with

$$
f=\frac{1}{g \theta}\left(1-\frac{1}{s}\right)
$$

( $s$ was defined in eq. (2.13)). The field strength $F_{i j}=\partial_{i} A_{j}-\partial_{j} A_{i}-i g\left[A_{i}, A_{j}\right]$ can be written more conveniently as

$$
F_{i j}=\frac{1}{s} \tilde{F}_{i j}+f_{i j}
$$

where

$$
f_{i j}=-\varepsilon_{i j} \frac{2 \pi k}{g} \frac{1}{\tilde{L}_{1} \tilde{L}_{2}}
$$

and

$$
\tilde{F}_{i j}=\partial_{i} \tilde{A}_{j}-\partial_{j} \tilde{A}_{i}-i \tilde{g}\left[\tilde{A}_{i}, \tilde{A}_{j}\right]
$$

where we have introduced the scaled charge,

$$
\tilde{g}=s g
$$

and we have used that

$$
\varepsilon_{i j}\left[x_{j},\right]=-i \theta \partial_{i} .
$$

Let see how does the field $A$ transforms under gauge transformations. Applying a gauge transformation to $(2.15)$ we have

$$
\begin{aligned}
A_{i}^{\prime} & =V\left(\tilde{A}_{i}+a_{i}\right) V+\frac{i}{g} V \partial_{i} V^{-1} \\
& =V \tilde{A}_{i} V+g f \varepsilon_{i j} V x^{j} V^{-1}+\frac{i}{g} V \partial_{i} V^{-1} .
\end{aligned}
$$

But using (2.22) we can rewrite the middle term as a derivative term plus $a_{i}$

$$
\begin{aligned}
A_{i}^{\prime} & =V \tilde{A}_{i} V-i \theta f V \partial_{i} V^{-1}+a_{i}+\frac{i}{g} V \partial_{i} V^{-1} \\
& =V \tilde{A}_{i} V+i \frac{1-g \theta f}{g} V \partial_{i} V^{-1}+a_{i} \\
& =V \tilde{A}_{i} V+\frac{i}{\tilde{g}} V \partial_{i} V^{-1}+a_{i} .
\end{aligned}
$$

Thus a gauge transformation on $A_{i}$ is equivalent to a gauge transformation on $\tilde{A}_{i}$ but with the scaled charge $\tilde{g}$ (and the field $a_{i}$ untransformed). 
We can summarize these results by stating that a gauge theory on the noncommutative torus $\mathcal{T}$ and with non-trivial boundary conditions (2.5), (2.6) is equivalent to a gauge theory on the scaled noncommutative torus $\tilde{\mathcal{T}}$, with periodic boundary conditions and with a scaled charge $\tilde{g}$.

Let us now solve the boundary condition equations for the Higgs field. A field $\phi(x)$ satisfying the boundary conditions (2.4) with the transition functions given in equation (2.8), can be decomposed as

$$
\phi(x, y)=\phi_{0}(x, y) \eta(x, y)
$$

where $\phi_{0}(x, y)$ is an arbitrary function periodic in the scaled torus $\tilde{\mathcal{T}}$ and $\eta(x, y)$ satisfy the same boundary conditions as $\phi(x, y)$. Then we just have to find a particular solution of

$$
\begin{aligned}
& \eta\left(x+L_{1}, y\right)=U_{1}(x, y) \eta(x, y) \\
& \eta\left(x, y+L_{2}\right)=U_{2}(x, y) \eta(x, y) .
\end{aligned}
$$

Inspired in the commutative case [6] let us consider a function $h(x, y)$ of the form

$$
h(x, y)=e^{i \alpha\{z, y\}}, \quad z=x+i y,
$$

where $\{z, y\}=z y+y z$ and $\alpha$ is determined by the condition

$$
\begin{aligned}
h\left(x+L_{1}, y\right) & =U_{1}(x, y) h(x, y) \\
& =e^{i \pi \omega L_{1} y} e^{i \alpha\{z, y\}} .
\end{aligned}
$$

Since $[z, y]=i \theta$, we can use the result (A.1) of the appendix to obtain

$$
U_{1}(x, y) h(x, y)=e^{i \alpha\{z+c, y\}}, \quad c=\frac{\theta \pi \omega L_{1}}{1-e^{-2 \theta \alpha}} .
$$

Then, equation (2.28) is solved if we chose

$$
\alpha=-\frac{1}{2 \theta} \log (1-\pi \omega \theta)=-\frac{1}{2 \theta} \log s .
$$

Now we compute

$$
U_{2}(x, y) h(x, y)=e^{-i \pi \omega L_{2} x} e^{i \alpha\{z, y\}}
$$

Using several times equations (A.1), (A.20), (A.21) and (A.22) of the appendix we get

$$
\begin{aligned}
U_{2}(x, y) h(x, y) & =e^{-k \pi L_{2} / L_{1}} e^{i \alpha\left\{z+i L_{2}, y-\tilde{L}_{2}\right\}} \\
& =e^{k \pi L_{2} / L_{1}} e^{i \alpha\left\{z+i L_{2}, y-L_{2}\right\}} e^{-i 2 \pi k z / L_{1}} \\
& =h\left(x, y+L_{2}\right) e^{k \pi L_{2} / L_{1}} e^{-i 2 \pi k z / L_{1}}
\end{aligned}
$$

(we used that $\left.\omega(1-\pi \omega \theta / 2)=k / L_{1} L_{2}\right)$. Then, $\eta(x, y)$ can be written as

$$
\eta(x, y)=h(x, y) \Theta(x, y)
$$


with $\Theta(x, y)$ satisfying

$$
\begin{aligned}
& \Theta\left(x+L_{1}, y\right)=\Theta(x, y) \\
& \Theta\left(x, y+L_{2}\right)=e^{k \pi L_{2} / L_{1}} e^{-i 2 \pi k z / L_{1}} \Theta(x, y) .
\end{aligned}
$$

In commutative space, a function that satisfies (2.34) is given by a product of Riemann $\theta_{3}$ functions

$$
\Theta(x, y)=\prod_{n=1}^{k} \theta_{3}\left(\pi\left(z+a_{n}\right) / L_{1} \mid i L_{1} / L_{2}\right),
$$

where

$$
\theta_{3}(z \mid \tau)=\sum_{n} e^{i \pi \tau n^{2}+2 i n z}
$$

and $a_{n}$ are arbitrary complex numbers satisfying

$$
\sum_{n=1}^{k} a_{n}=0
$$

(the function $\Theta(x, y)$ has $k$ zeros at the points $a_{i}+\left(L_{1}+i L_{2}\right) / 4$ ).

However, since the theta functions depend only on one variable, we can replace the standard product with the noncommutative product, as they both coincide. Then, eq. (2.35) is the solution of (2.34) in noncommutative space. Thus,

$$
\eta(x, y)=e^{i \alpha\{z, y\}} \prod_{n=1}^{k} \theta_{3}\left(\pi\left(z+a_{n}\right) / L_{1} \mid i L_{1} / L_{2}\right)
$$

with

$$
\alpha=-\frac{1}{2 \theta} \log s
$$

In the limit $\theta \rightarrow 0$ this function coincides with the one obtained in the commutative case (see $[6]$ ). For the special case of $k=1$ we have

$$
\eta(x, y)=e^{i \alpha\{z, y\}} \theta_{3}\left(\pi z / L_{1} \mid i L_{1} / L_{2}\right) .
$$

In order to discuss the dynamics through the introduction of the action and the energy of our model, we have to define an appropriate trace (or integral) on the noncommutative torus. Calling $\mathcal{A}_{\theta}$ the space of functions defined on $\mathcal{T}$, a generic periodic function $f(x, y)$ can be written in the form

$$
f(x, y)=\sum_{m, n} f_{m n} \exp \left(i m \frac{x}{L_{1}}\right) \exp \left(i n \frac{y}{L_{2}}\right)
$$

and then one can formally define integration in $\mathcal{A}_{\theta}$, which we shall call trace $\operatorname{Tr}$, as follows

$$
\operatorname{Tr} f(x, y)=f_{00} L_{1} L_{2}
$$

which in turns defines an integral over $\mathcal{T}$. This operation satisfies $\operatorname{Tr}(f g)=\operatorname{Tr}(g f)$ and reduces in the commutative limit to the standard integral on $\mathcal{T}$. 
We have defined in (2.42) the integration in the noncommutative torus of strictly periodic functions $f(x, y)$. However the definition has to be corrected when the integrand satisfies twisted boundary conditions $[9,10]$. We discuss this issue in detail in appendix 3 and here give a brief summary. Consider a function $f(x, y)$ that satisfies twisted boundary conditions in the adjoint section (as it is the case of $F_{i j}$ for example)

$$
\begin{aligned}
& f\left(x+L_{1}, y\right)=U_{1}(x, y) f(x, y) U_{1}^{-1}(x, y) \\
& f\left(x, y+L_{2}\right)=U_{2}(x, y) f(x, y) U_{2}^{-1}(x, y) .
\end{aligned}
$$

Then, using $(2.10)$ we see that $f(x, y)$ is in fact periodic in the scaled torus $\tilde{\mathcal{T}}$. So the natural integration measure for the function $f(x, y)$ is on the scaled torus $\tilde{\mathcal{T}}$, that is

$$
I[f]=\operatorname{Tr}_{\tilde{\mathcal{T}}} f .
$$

It can be shown that this definition is crucial if we want to preserve the cyclic property of the integral (trace) which is essential in order to derive the equations of motion. Consider for example two functions $\phi_{1}(\vec{x})$ and $\phi_{2}(\vec{x})$ that have nontrivial boundary conditions

$$
\begin{aligned}
& \phi_{i}\left(x+L_{1}, y\right)=U_{1}(x, y) \phi_{i}(x, y) \\
& \phi_{i}\left(x, y+L_{2}\right)=U_{2}(x, y) \phi_{i}(x, y), \quad i=1,2 .
\end{aligned}
$$

Then the product

$$
\phi_{1}(\vec{x}) \phi_{2}^{\dagger}(\vec{x})
$$

is strictly periodic in the torus $\mathcal{T}$, but the transpose product,

$$
\phi_{2}^{\dagger}(\vec{x}) \phi_{1}(\vec{x})
$$

satisfy nontrivial boundary conditions in the adjoint, so it is periodic in the scaled torus $\tilde{\mathcal{T}}$. Nonetheless, as we show in the appendix, the cyclic property of the integral is still valid provided we integrate the first function in $\mathcal{T}$ and the second one in $\tilde{\mathcal{T}}$

$$
\operatorname{Tr}_{\mathcal{T}}\left(\phi_{1}(\vec{x}) \phi_{2}^{\dagger}(\vec{x})\right)=\operatorname{Tr}_{\tilde{\mathcal{T}}}\left(\phi_{2}^{\dagger}(\vec{x}) \phi_{1}(\vec{x})\right) .
$$

That is, the cyclic property is preserved with the above definition.

\section{The Maxwell-Higgs model}

We shall consider here a U(1) gauge field coupled to a Higgs scalar defined on the noncommutative torus. Dynamics of the model is governed by the lagrangian

$$
L=-\frac{1}{4} F_{\mu \nu} F^{\mu \nu}+\left(D_{\mu} \Phi\right)^{\dagger}\left(D^{\mu} \Phi\right)-\lambda\left(\Phi^{\dagger} \Phi-\phi_{0}^{2}\right)^{2} .
$$

We are interested in static configurations so that the energy can be written in the form ${ }^{1}$

$$
E=\operatorname{Tr}\left(\frac{1}{4} F_{i j} F_{i j}+\left(D_{i} \Phi\right)^{\dagger}\left(D_{i} \Phi\right)+\lambda\left(\Phi^{\dagger} \Phi-\phi_{0}^{2}\right)^{2}\right) .
$$

\footnotetext{
${ }^{1}$ In this expression we are mixing covariantly periodic terms $\left(F_{i j} F_{i j}\right)$ with strictly periodic terms, $\left(\left(D_{i} \Phi\right)^{\dagger}\left(D_{i} \Phi\right)\right.$ and $\left.\left(\Phi^{\dagger} \Phi-\phi_{0}^{2}\right)^{2}\right)$, so according to the previous discussion on integration, the integrals have to be defined in their appropriate domains. Note however, that we can convert the periodic terms into covariantly periodic ones by using property (2.48), and thus, the whole lagrangian or energy have to be integrated in the same domain, the scaled torus $\tilde{\mathcal{T}}$.
} 
Here $D_{i} \Phi=\partial_{i} \Phi-i g A_{i} \Phi$ is the covariant derivative and $F_{i j}$ is the electromagnetic tensor. Notice that, via the covariant derivative, we are choosing for definiteness a Higgs-gauge coupling which corresponds to the fundamental representation (other choices are possible).

As in the commutative case, the energy can be rewritten using the Bogomolny trick as,

$$
\begin{aligned}
E=\operatorname{Tr}( & \frac{1}{2}\left|D_{i} \Phi-i \gamma \varepsilon_{i j} D_{j} \Phi\right|^{2}+\frac{1}{4}\left(F_{i j}-\gamma g \varepsilon_{i j}\left(\Phi \Phi^{\dagger}-\phi_{0}^{2}\right)\right)^{2}+ \\
& \left.+\left(\lambda-\frac{g^{2}}{2}\right)\left(\Phi^{\dagger} \Phi-\phi_{0}\right)^{2}-\gamma \frac{g}{2} \phi_{0}^{2} \varepsilon_{i j} F_{i j}+\text { total derivative }\right)
\end{aligned}
$$

where $\gamma= \pm 1$

The BPS equation corresponding to a bound of the energy when $\lambda=g^{2} / 2$,

$$
E \geq-\gamma \frac{g}{2} \phi_{0}^{2} \operatorname{Tr}_{\tilde{\mathcal{T}}} \varepsilon_{i j} F_{i j}
$$

then read,

$$
\begin{aligned}
D_{i} \Phi-i \gamma \varepsilon_{i j} D_{j} \Phi & =0 \\
F_{i j}-\gamma g \varepsilon_{i j}\left(\Phi \Phi^{\dagger}-\phi_{0}^{2}\right) & =0 .
\end{aligned}
$$

Setting for definiteness $\gamma=-1$ and using (2.15)-(2.18), we can write the BPS equations as

$$
\begin{aligned}
& \tilde{F}_{12}=\tilde{g}\left(\Phi \Phi^{\dagger}-\left(\phi_{0}^{2}-\frac{2 \pi k}{g^{2} \tilde{L}_{1} \tilde{L}_{2}}\right)\right) \\
& \tilde{D}_{\bar{z}} \Phi+\frac{\pi \omega}{2} \Phi z=0,
\end{aligned}
$$

where $z=x+i y$.

Since the fields $\tilde{A}$ are periodic in the scaled torus $\tilde{\mathcal{T}}$, the total flux of $\tilde{F}_{i j}$ on $\tilde{\mathcal{T}}$ vanishes (see equation (2.42)) and then we have

$$
\Phi=\operatorname{Tr}_{\tilde{\mathcal{T}}} F_{12}=\operatorname{Tr}_{\tilde{\mathcal{T}}} f_{12}=-\frac{2 \pi k}{g} .
$$

Bogomolny equations (3.7)-(3.8) have the particular solution

$$
\widetilde{A}=\Phi=0
$$

provided the area of the torus and the Higgs vev are related according to

$$
\phi_{0}^{2}=\frac{2 \pi k}{g^{2} \tilde{L}_{1} \tilde{L}_{2}} .
$$

In the $\theta \rightarrow 0$ commutative limit this solution reproduces the so called Bradlow solution [11] on the torus. Moreover, as in the commutative case [6], solution (3.10)-(3.11) could then be used as a starting point to obtain new solutions with non-vanishing $\tilde{A}$ and $\Phi$, by an appropriate expansion. 
In order to search for general solutions to eqs. (3.7)-(3.8) it will be convenient to parametrize the fields as

$$
\begin{aligned}
\tilde{A}_{\bar{z}} & =\frac{i}{\tilde{g}} M^{-1} \partial_{\bar{z}} M+\tilde{A}_{\bar{z}}^{0} \\
\Phi & =M^{-1} \chi,
\end{aligned}
$$

where $M$ is a complex (non unitary) function periodic in $\tilde{\mathcal{T}}, \tilde{A}_{\bar{Z}}^{0}$ is a constant field, and $\chi$ has the same periodicity as $\Phi$. The BPS equation (3.8) then becomes,

$$
\partial_{\bar{z}} \chi-i \tilde{g} \tilde{A}_{\bar{z}}^{0} \chi+\frac{\pi \omega}{2} \chi z=0 \text {. }
$$

As we showed previously in equation (2.25), the function $\chi$ can be factorized as

$$
\chi(x, y)=\chi_{0}(x, y) \eta(x, y),
$$

where $\eta$ carries the non trivial boundary conditions (see eq. (2.38)) and $\chi_{0}$ is periodic in $\tilde{\mathcal{T}}$. Replacing (3.15) in (3.14) we get

$$
\left(\partial_{\bar{z}} \chi_{0}-i \tilde{g} \tilde{A}_{\bar{z}}^{0} \chi_{0}\right) \eta+\chi_{0}\left(\partial_{\bar{z}} \eta+\frac{\pi \omega}{2} z\right)=0 .
$$

To compute $\partial_{\bar{z}} \eta$ we first use equations (A.21) and (A.22) of the appendix to rewrite

$$
\eta(x, y)=e^{-\alpha\{z, \bar{z}\}} e^{\pi k z^{2} / 2 L_{1} L_{2}} \Theta(z),
$$

where $\Theta$, given in equation (2.35), in only function of $z$. Thus the problem reduces to compute the derivative with respect to $\bar{z}$ of $e^{-\alpha\{z, \bar{z}\}}$. Using that

$$
\partial_{\bar{z}}=\frac{1}{2 \theta}[z,]
$$

and that $[z, \bar{z}]=2 \theta$ we can show that

$$
\partial_{\bar{z}} e^{-\alpha\{z, \bar{z}\}}=-\frac{\pi \omega}{2} e^{-\alpha\{z, \bar{z}\}} z
$$

and then the second term of equation (3.16) vanishes. So, the BPS equation (3.8) reduces to

$$
\partial_{\bar{z}} \chi_{0}-i \tilde{g} \tilde{A}_{\bar{z}}^{0} \chi_{0}=0
$$

with solution

$$
\chi_{0}=N e^{i \widetilde{g}\left(i \tilde{A}_{\tilde{z}}^{0} \bar{z}+\tilde{A}_{z}^{0} z\right)},
$$

where $N$ is a normalization factor. Periodicity of $\chi_{0}$ requires that $\tilde{A}^{0}$ has the form

$$
\tilde{A}_{z}^{0}=\frac{\pi}{\tilde{g}}\left(\frac{n_{0}}{\tilde{L}_{1}}+i \frac{m_{0}}{\tilde{L}_{2}}\right)
$$

with integers $n_{0} \mathrm{y} m_{0}$. In commutative space this particular form of $A_{0}$ is a pure gauge and thus can be simply gauged away. In noncommutative space this is also the case with the proviso that the gauge transformation will also transform the non-trivial part of the field $\tilde{A}$ (equation (3.12)). However the effect of the transformation will be only a shift in the coordinates of the fields. So, without losing generality we can make $m_{0}=n_{0}=0$. 
Concerning the BPS equation eq. (3.8), one has first to write the field $\tilde{F}_{12}$ in terms of the variables $M$ defined in eq. (3.12). Clearly the gauge invariant variables are to be defined from the combination

$$
H=M M^{\dagger}
$$

so that one should be able to write the Bogomolny equations in terms of $H$. Since $\tilde{F}_{12}$ is not gauge invariant but covariant, one can not write it only in terms of $H$; indeed a straightforward computation gives

$$
\tilde{F}_{z \bar{z}}=\frac{i}{\tilde{g}} M^{-1} H \partial_{z}\left(H^{-1} \partial_{\bar{z}} H\right) M^{\dagger-1}
$$

Substituting this expression, and that for $\Phi$ given by eq. (3.13) in the Bogomolny equation leads to

$$
H \partial_{z}\left(H^{-1} \partial_{\bar{z}} H\right)=\frac{1}{2} \tilde{g}^{2}\left(\chi \chi^{\dagger}-\mu_{0}^{2} H\right)
$$

where $\chi$ is given in equations (3.15), (3.22) and

$$
\mu_{0}^{2}=\phi_{0}^{2}-\frac{2 \pi k}{g^{2} \tilde{L}_{1} \tilde{L}_{2}} .
$$

In order to make further progress to find solutions of eq. (3.25) one has in principle to resort to numerical techniques as it is already the case for $\theta=0$.

\section{Non abelian extension and discussion}

It should be possible to extend most of our results to the case of (appropriate) non abelian gauge groups. As it is well known, consistency of noncommutative theories requires to work with $\mathrm{U}(N)$ groups and not $\mathrm{SU}(N)[12]$. One can then consider a $\mathrm{U}(2) \times \mathrm{U}(1)$ model as a first step in the study of vortex solutions in a noncommutative version of the standard model, along the lines of Ref.[4] for the commutative case.

Consider then the energy for static configurations,

$$
E=\operatorname{Tr}_{\mathcal{T}}\left(\frac{1}{2} \operatorname{tr}\left(W_{i j} W_{i j}\right)+\frac{1}{4} \operatorname{tr}\left(B_{i j} B_{i j}\right)+\left(D_{i} \Phi\right)^{\dagger}\left(D_{i} \Phi\right)+\lambda\left(\Phi^{\dagger} \Phi-\phi_{0}^{2}\right)^{2}\right)
$$

where the U(2) gauge fields are defined as

$$
W_{i}=W_{i}^{a} \lambda^{a}, \quad \lambda^{0}=\frac{1}{2} I, \quad \lambda^{k}=\frac{1}{2} \sigma^{k}
$$

$B_{i}$ is a $\mathrm{U}(1)$ gauge field, $\Phi$ is a Higgs field in the fundamental representation of $\mathrm{U}(2)$ and the covariant derivatives and field strengths are defined as

$$
\begin{gathered}
D_{i} \Phi=\partial_{i} \Phi-i g W_{i} \Phi+i \frac{g^{\prime}}{2} \Phi B_{i} \\
W_{i j}=\partial_{i} W_{j}-\partial_{j} W_{i}+i g\left[W_{i}, W_{j}\right], \quad B_{i j}=\partial_{i} B_{j}-\partial_{j} B_{i}+i g^{\prime}\left[B_{i}, B_{j}\right] .
\end{gathered}
$$


Notice that the covariant derivative is defined so that it acts from the left for the $U(2)$ group and from the right for the $\mathrm{U}(1)$ one. The appropriate way to write perfect a square à la Bogomolny for the Higgs covariant derivative is in this case

$$
\begin{aligned}
\left|D_{i} \Phi\right|^{2}= & \left|D_{i} \Phi-i \gamma \varepsilon_{i j} D_{j} \Phi\right|^{2}-\gamma g \operatorname{tr}\left(\varepsilon_{i j} \Phi^{\dagger} W_{i j} \Phi\right)+\gamma \frac{g^{\prime}}{2}\left(\Phi^{\dagger} \Phi\right) \varepsilon_{i j} B_{i j}+ \\
& + \text { divergence }
\end{aligned}
$$

leading to the following expression for the energy:

$$
\begin{aligned}
E=\operatorname{Tr}( & \frac{1}{2}\left|D_{i} \Phi-i \gamma \varepsilon_{i j} D_{j} \Phi\right|^{2}+\frac{1}{2} \operatorname{tr}\left(W_{i j}-\gamma \varepsilon \frac{g}{2} \Phi \Phi^{\dagger}\right)^{2}+ \\
& +\frac{1}{4}\left(B_{i j}+\gamma \frac{g^{\prime}}{2} \varepsilon_{i j}\left(\Phi^{\dagger} \Phi-\mu^{2} \phi_{0}^{2}\right)\right)^{2}+\gamma \frac{g^{\prime}}{2} \mu^{2} \phi_{0}^{2} \varepsilon_{i j} B_{i j}+ \\
& +\left(\lambda-\frac{g^{2}}{4}-\frac{g^{\prime 2}}{8}\right)\left(\Phi^{\dagger} \Phi-\phi_{0}\right)^{2}-\left(\frac{g^{2}}{2}+\frac{g^{\prime 2}}{4}\left(1-\mu^{2}\right)\right) \Phi^{\dagger} \Phi \phi_{0}^{2}+ \\
& \left.+\left(\frac{g^{2}}{2}+\frac{g^{\prime 2}}{4}\left(1-\mu^{4}\right)\right) \phi_{0}^{4}\right) .
\end{aligned}
$$

Then, if we choose

$$
\mu^{2}=1+2 \frac{g^{2}}{g^{\prime 2}} \quad \lambda=\frac{g^{2}}{4}+\frac{g^{\prime 2}}{8}
$$

the energy is bounded as

$$
E \geq \gamma g^{\prime} \mu^{2} \phi_{0}^{2} \Phi_{B}-\mu^{2}\left(\mu^{2}-1\right) \phi_{0}^{4} A,
$$

where $\Phi_{B}$ is the flux of the $B$ field and $A$ is the area of the torus.

The bound is attained when the following BPS equations are satisfied,

$$
\begin{aligned}
D_{i} \Phi-i \gamma \varepsilon_{i j} D_{j} \Phi & =0 \\
B_{i j}+\gamma \frac{g^{\prime}}{2} \varepsilon_{i j}\left(\Phi^{\dagger} \Phi-\mu^{2} \phi_{0}^{2}\right) & =0 \\
W_{i j}-\gamma \varepsilon_{i j} \frac{g}{2} \Phi \Phi^{\dagger} & =0 .
\end{aligned}
$$

As in the commutative space case [4]-[6], the bound has a topological component, proportional to the $B$ flux and a geometrical part, proportional to the area of the torus. The non-commutative nature of space and the extra $\mathrm{U}(1)$ factor associated to the $\mathrm{U}(2)$ group renders nevertheless, the analysis of the solutions of these equations considerably more involved.

Let us end our work by summarizing our main results. We have analyzed periodic configurations of matter and gauge fields in non commutative space. We have discussed in detail how as a result of coordinate non commutativity, the region of periodicity of gauge invariant and gauge covariant quantities may differ, a property that has to be kept in mind in order to obtain consistent results. In this work, we have focussed mainly in the abelian Maxwell Higgs model, where we have been able to obtain BPS equations whose vortex solutions also solve the Euler Lagrange equations. We have presented a particular solution 
to these equations which, in the $\theta \rightarrow 0$ commutative limit corresponds the Bradlow solution on the commutative torus. In the general case, we were able to reduce the problem of the two coupled BPS equation to that of equation (3.25), which in principle should be solved using numerical techniques, as it is the case for the commutative torus [6].

We believe that the generalization to non-abelian models will not present major difficulties. As a particular example and as a first step in this direction, we have shown how the BPS equations of a $U(2) \times U(1)$, a simplified version of the Standard Model in non commutative space, are obtained. Of course, the noncommutative character both of the space and the gauge group makes the obtention of explicit solutions much more complicated but a more detailed analysis should reveal the existence of Z-vortex arrays (possibly with the presence of charged mesons condensates) as it is the case in ordinary space [4]. We hope to report on this issues in a future publication.

\section{Acknowledgments}

This work was partially supported by UNLP, CICBA, CONICET, ANPCYT (PICT grant 03-05179) Argentina and ECOS-Sud Argentina-France collaboration (grant A01E02). F.A.S. wishes to thank the Marie de Paris for the support of a Senior Fellowship during part of this work.

\section{A. A useful result}

We prove here a helpful result that was used extensively throughout the paper:

Lemma. Let $A$ and $B$ be two operators such that $[A, B]=i \mu A$ where $\mu$ is an arbitrary constant, then

$$
e^{i A} e^{i B}=e^{i(f(\mu) A+B)}
$$

where

$$
f(\mu)=\frac{\mu}{e^{\mu}-1} .
$$

Proof. We write

$$
e^{i A} e^{i B}=e^{i C} .
$$

First we notice that a quick look at the Campbell-Baker-Hausdorff formula

$$
e^{A} e^{B}=e^{A+B+\frac{1}{2}[A, B]+\frac{1}{12}([A,[A, B]]+[B,[B, A]])+\cdots}
$$

reveals that $C$ must be of the form

$$
C=f(\mu) A+B
$$

since any arbitrary nested commutator with $[A, B]$ will give, either zero or something proportional to $A$. So the problem reduces to find the function $f(\mu)$. 
Consider now the function

$$
U(s)=e^{i s A} e^{i s B}
$$

we have that

$$
\frac{d U}{d s} U^{-1}=i\left(A+U B U^{-1}\right) .
$$

But

$$
\begin{aligned}
e^{i s A} B e^{-i s A} & =B+i s[A, B] \\
& =B-s \mu A
\end{aligned}
$$

since higher order commutators vanish. Thus we have

$$
\frac{d U}{d s}=i((1-s \mu) A+B) U .
$$

Now we write according to (A.3) and (A.5)

$$
U(s)=e^{i C(s)}, \quad C(s)=s(f(s \mu) A+B) .
$$

We have

$$
\frac{d U}{d s}=\sum_{n=0}^{\infty} \frac{i^{n}}{n !} \frac{d C(s)^{n}}{d s}
$$

but

$$
\begin{aligned}
\frac{d C(s)^{n}}{d s} & =\sum_{p=0}^{n-1} C^{p} \frac{d C(s)}{d s} C^{n-p} \\
& =s^{-1} n C(s)+\mu f^{\prime}(s \mu) \sum_{p=0}^{n-1} C^{p} A C^{n-p} .
\end{aligned}
$$

Now we notice that

$$
\begin{aligned}
C(s) A & =A C(s)+[C(s), A] \\
& =A(C(s)-i s \mu)
\end{aligned}
$$

and applying successively this result we have

$$
C(s)^{p} A=A(C(s)-i s \mu)^{p} .
$$

Replacing this result back in (A.12) and then in (A.11) we get

$$
\frac{d U}{d s}=i s^{-1} C(s) U+\mu f^{\prime}(s \mu) A \sum_{n=0}^{\infty} \frac{i^{n}}{n !} \sum_{p=0}^{n-1}(C(s)-i s \mu)^{p} C(s)^{n-p-1} .
$$

The sum in $p$ is a geometric sum, so it can be easily performed. It gives

$$
\sum_{p=0}^{n-1}(C(s)-i s \mu)^{p} C(s)^{n-p-1}=(i s \mu)^{-1}\left(C(s)^{n}-(C(s)-i s \mu)^{n}\right)
$$


and substituting this result in (A.15) we get

$$
\begin{aligned}
\frac{d U}{d s} & =i s^{-1} C(s) U+\mu f^{\prime}(s \mu) A \sum_{n=0}^{\infty} \frac{i^{n}}{n !}(i s \mu)^{-1}\left(C(s)^{n}-(C(s)-i s \mu)^{n}\right) \\
& =i s^{-1} C(s) U-i s^{-1} f^{\prime}(s \mu) A\left(e^{i C(s)}-e^{i(C(s)-i s \mu)}\right) \\
& =i\left(\left(f-f^{\prime}\left(1-e^{s \mu}\right)\right) A+B\right) U .
\end{aligned}
$$

Finally, comparing this equation with (A.9) we have the following differential equation for $f$

$$
f-f^{\prime}\left(1-e^{s \mu}\right)=1-s \mu .
$$

The solution (with the initial condition $f(0)=1$, as can be deduced from the series expansion of (A.6)) is

$$
f(s \mu)=\frac{s \mu}{e^{s \mu}-1}
$$

and evaluating in $s=1$ we get the desired result.

Taking the inverse of expression (A.1) (and rescaling the fields and $\mu$ ) we have the equivalent result:

$$
e^{i B} e^{i A}=e^{i(g(\mu) A+B)}, \quad g(\mu)=\frac{\mu}{1-e^{-\mu}} .
$$

Similarly we can prove,

$$
e^{i(A+B)}=e^{i h(\mu) A} e^{i B}, \quad h(\mu)=\frac{e^{\mu}-1}{\mu}
$$

and

$$
e^{i(A+B)}=e^{i B} e^{i k(\mu) A}, \quad k(\mu)=\frac{1-e^{-\mu}}{\mu} .
$$

In all cases

$$
[A, B]=i \mu A \text {. }
$$

\section{B. Cyclic property of the integral}

We will show below that the cyclic property of the integral is valid whenever one defines the integration on the appropriate torus.

First, from the definition of the integration on the torus, it is straightforward to see that for strictly periodic functions $f(x, y)$ and $g(x, y)$, the cyclic property holds

$$
\int_{\mathcal{T}} f g=\int_{\mathcal{T}} g f .
$$

Consider now two functions $\phi_{1}(x, y)$ and $\phi_{2}(x, y)$ satisfying the non-trivial periodic conditions

$$
\begin{aligned}
\phi_{i}\left(x+L_{1}, y\right) & =U_{1}(x, y) \phi_{i}(x, y) \\
\phi_{i}\left(x, y+L_{2}\right) & =U_{2}(x, y) \phi_{i}(x, y), \quad i=1,2 .
\end{aligned}
$$


The product

$$
w_{1}(x, y)=\phi_{1}^{\dagger}(x, y) \phi_{2}(x, y)
$$

is periodic on the torus $\mathcal{T}$. On the other hand the reversed product

$$
w_{2}(x, y)=\phi_{2}(x, y) \phi_{1}^{\dagger}(x, y)
$$

is periodic in the scaled torus $\tilde{\mathcal{T}}$ (2.43). Thus, following the definition of integral on the noncommutative torus, $w_{1}$ must be integrated on $\mathcal{T}$ and $w_{2}$ on $\tilde{\mathcal{T}}$. We will show that this definition satisfies

$$
\int_{\mathcal{T}} w_{1}(x, y)=\int_{\tilde{\mathcal{T}}} w_{2}(x, y)
$$

Let us consider, for simplicity, the case $k=1$. As we showed previously, the functions $\phi_{1}$ and $\phi_{2}$ can be decomposed as

$$
\begin{aligned}
& \phi_{1}(x, y)=\phi_{1}^{0}(x, y) \eta(x, y) \\
& \phi_{2}(x, y)=\phi_{2}^{0}(x, y) \eta(x, y),
\end{aligned}
$$

where $\phi_{i}^{0}(x, y), i=1,2$ are periodic in $\tilde{\mathcal{T}}$ and

$$
\eta(x, y)=e^{i \alpha\{z, y\}} \theta_{3}\left(\frac{\pi z}{L_{1}} \mid \frac{i L_{1}}{L_{2}}\right), \quad \alpha=-\frac{1}{2 \theta} \log s .
$$

Then

$$
\begin{aligned}
& w_{1}(x, y)=\eta^{\dagger}(x, y) \phi_{1}^{0 \dagger}(x, y) \phi_{2}^{0}(x, y) \eta(x, y) \\
& w_{2}(x, y)=\phi_{2}^{0}(x, y) \eta(x, y) \eta^{\dagger}(x, y) \phi_{1}^{0 \dagger}(x, y)
\end{aligned}
$$

Consider first the integral

$$
I_{1}=\int_{\mathcal{T}} w_{1}(x, y)=\int_{\mathcal{T}} \eta^{\dagger}(x, y) \phi_{1}^{0 \dagger}(x, y) \phi_{2}^{0}(x, y) \eta(x, y) .
$$

Since $\phi_{1}^{0 \dagger}(x, y) \phi_{2}^{0}(x, y)$ is periodic in $\tilde{\mathcal{T}}$, without loss of generality we can replace it by

$$
e^{i 2 \pi\left(n x / \tilde{L}_{1}+m y / \tilde{L}_{2}\right)}, \quad n, m \in \mathbb{Z} .
$$

Consider now the product

$$
\gamma(x, y)=\eta^{\dagger}(x, y) e^{i 2 \pi\left(n x / \tilde{L}_{1}+m y / \tilde{L}_{2}\right)} \eta(x, y) .
$$

It can be easily shown that for any function $f(x, y)$

$$
f(x, y) e^{i 2 \pi\left(n x / \tilde{L}_{1}+m y / \tilde{L}_{2}\right)}=e^{i 2 \pi\left(n x / \widetilde{L}_{1}+m y / \tilde{L}_{2}\right)} f\left(x-t_{1}, y+t_{2}\right),
$$

where

$$
t_{1}=2 \pi m \frac{\theta}{\tilde{L}_{2}}, \quad t_{2}=2 \pi n \frac{\theta}{\tilde{L}_{1}} .
$$


Thus

$$
\begin{aligned}
\gamma(x, y)= & e^{i 2 \pi\left(n x / \tilde{L}_{1}+m y / \tilde{L}_{2}\right)} \eta^{\dagger}\left(x-t_{1}, y+t_{2}\right) \eta(x, y) \\
= & \sum_{p} \sum_{q} e^{i 2 \pi\left(n x / \tilde{L}_{1}+m y / \tilde{L}_{2}\right)} e^{-\pi L_{2} / L_{1} p^{2}+i 2 \pi p\left(\bar{z}-t_{1}-i t_{2}\right) / L_{1}} \times \\
& \times e^{-i \alpha\left\{\bar{z}-t_{1}-i t_{2}, y+t_{2}\right\}} e^{i \alpha\{z, y\}} e^{-\pi L_{2} / L_{1} q^{2}+i 2 \pi q\left(z-t_{1}+i t_{2}\right) / L_{1}} .
\end{aligned}
$$

Next we have to expand this expression in Fourier modes

$$
\gamma(x, y)=\sum_{p, q} \gamma_{p q} e^{i 2 \pi\left(p x / \tilde{L}_{1}+q y / \tilde{L}_{2}\right)}
$$

and keep the coefficient $\gamma_{00}$. Using several times the identities (A.1), (A.20), (A.21), and (A.22), and after a straightforward but long computation, we get

$$
\gamma_{00}= \begin{cases}0 & \text { if } m \text { is even } \\ s \sqrt{2 L_{1} / L_{2}} e^{-\pi^{2}\left(L_{1}^{2} m^{2}+L_{2} n^{2}\right) / 2 \tilde{L}_{1} \tilde{L}_{2}} & \text { if } m \text { is odd } .\end{cases}
$$

Then

$$
I_{1}=\int_{\mathcal{T}} \gamma(x, y)=L_{1} L_{2} \gamma_{00}
$$

Now consider the integral

$$
I_{2}=\int_{\tilde{\mathcal{T}}} w_{2}(x, y)=\int_{\tilde{T}} \phi_{2}^{0}(x, y) \eta(x, y) \eta^{\dagger}(x, y) \phi_{1}^{0 \dagger}(x, y) .
$$

Since the product $\eta(x, y) \eta^{\dagger}(x, y)$ is periodic in the torus $\tilde{\mathcal{T}}$, as well as $\phi_{1}^{0 \dagger}(x, y)$ and $\phi_{2}^{0}(x, y)$, we can rewrite $I_{2}$ as

$$
I_{2}=\int_{\tilde{\mathcal{T}}} \phi_{1}^{0 \dagger}(x, y) \phi_{2}^{0}(x, y) \eta(x, y) \eta^{\dagger}(x, y)
$$

and again replace $\phi_{1}^{0 \dagger}(x, y) \phi_{2}^{0}(x, y)$ by $e^{i 2 \pi\left(n x / \tilde{L}_{1}+m y / \tilde{L}_{2}\right)}$.

First we compute

$$
\begin{aligned}
\eta(x, y) \eta^{\dagger}(x, y)= & \sum_{p} \sum_{q} e^{i \alpha\{z, y\}} e^{-\pi L_{2} / L_{1} p^{2}+i 2 \pi p z / L_{1}} \times \\
& \times e^{-\pi L_{2} / L_{1} q^{2}+i 2 \pi q \bar{z} / L_{1}} e^{-i \alpha\{\bar{z}, y\}} .
\end{aligned}
$$

After another long computation, using the identities (A.1), (A.20), (A.21), and (A.22), we can write

$$
\eta(x, y) \eta^{\dagger}(x, y)=s^{-1} \sqrt{\frac{2 L_{1}}{L_{2}}} \sum_{p, q} e^{-\pi\left(L_{2}^{2} p^{2}+4 L_{1}^{2} q^{2}\right) / 2 s^{2}} e^{i 2 \pi\left(p x / \tilde{L}_{1}+2 q y / \tilde{L}_{2}\right)} .
$$

Thus, $\delta_{00}$, the $(0,0)$ Fourier mode of the product

$$
\delta(x, y)=e^{i 2 \pi\left(n x / \tilde{L}_{1}+m y / \tilde{L}_{2}\right)} \eta(x, y) \eta^{\dagger}(x, y)
$$


is given by

$$
\delta_{00}= \begin{cases}0 & \text { if } m \text { is even } \\ s^{-1} \sqrt{2 L_{1} / L_{2}} e^{-\pi^{2}\left(L_{1}^{2} m^{2}+L_{2} n^{2}\right) / 2 \tilde{L}_{1} \tilde{L}_{2}} & \text { if } m \text { is odd } .\end{cases}
$$

Notice that $\delta_{00}$ differs from $\gamma_{00}$ in a factor $s^{2}$ which precisely the relation between the area of the two torus. The integral is

$$
I_{2}=\int_{\tilde{\mathcal{T}}} w_{2}(x, y)=\tilde{L}_{1} \tilde{L}_{2} \delta_{00}=L_{1} L_{2} s^{2} \delta_{00}=L_{1} L_{2} \gamma_{00} .
$$

So

$$
\int_{\mathcal{T}} \phi_{1}^{\dagger}(x, y) \phi_{2}(x, y)=\int_{\tilde{\mathcal{T}}} \phi_{2}(x, y) \phi_{1}^{\dagger}(x, y)
$$

\section{References}

[1] M.R. Douglas and N.A. Nekrasov, Noncommutative field theory, Rev. Mod. Phys. 73 (2001) 977 [hep-th/0106048].

[2] F.A. Schaposnik, Proceedings of the Second Conference on Fundamental Interactions, Pedra Azul, Brasil, 2004; Three lectures on noncommutative field theories, hep-th/0408132.

[3] D.P. Jatkar, G. Mandal and S.R. Wadia, Nielsen-Olesen vortices in noncommutative abelian Higgs model, JHEP 09 (2000) 018 [hep-th/0007078];

A.P. Polychronakos, Flux tube solutions in noncommutative gauge theories, Phys. Lett. B 495 (2000) 407 [hep-th/0007043];

J.A. Harvey, P. Kraus and F. Larsen, Exact noncommutative solitons, JHEP 12 (2000) 024 [hep-th/0010060];

D. Bak, Exact multi-vortex solutions in noncommutative abelian-Higgs theory, Phys. Lett. B 495 (2000) 251 [hep-th/0008204];

G.S. Lozano, E.F. Moreno and F.A. Schaposnik, Nielsen-Olesen vortices in noncommutative space, Phys. Lett. B 504 (2001) 117 [hep-th/0011205]; Self-dual Chern-Simons solitons in noncommutative space, JHEP 02 (2001) 036 [hep-th/0012266];

A. Khare and M.B. Paranjape, Solitons in 2+1 dimensional non-commutative Maxwell Chern-Simons Higgs theories, JHEP 04 (2001) 002 [hep-th/0102016];

D. Bak, K.M. Lee and J.H. Park, Noncommutative vortex solitons, Phys. Rev. D 63 (2001) 125010 [hep-th/0011099];

R. Gopakumar, M. Headrick and M. Spradlin, On noncommutative multi-solitons, Commun. Math. Phys. 233 (2003) 355 [hep-th/0103256];

D. Tong, The moduli space of noncommutative vortices, J. Math. Phys. 44 (2003) 3509 [hep-th/0210010];

A. Hanany and D. Tong, Vortices, instantons and branes, JHEP 07 (2003) 037 [hep-th/0306150];

G.S. Lozano, E.F. Moreno, M.J. Rodriguez and F.A. Schaposnik, Non BPS noncommutative vortices, JHEP 11 (2003) 049 [hep-th/0309121].

[4] G. Bimonte and G. Lozano, Z flux line lattices and selfdual equations in the standard model, Phys. Rev. D 50 (1994) 6046 [hep-th/9403128].

[5] P.A. Shah and N.S. Manton, Thermodynamics of vortices in the plane, J. Math. Phys. 35 (1994) 1171 [hep-th/9307165]. 
[6] A. Gonzalez-Arroyo and A. Ramos, Expansion for the solutions of the Bogomolny equations on the torus, JHEP 07 (2004) 008.

[7] A. González-Arroyo, Yang-Mills fields on the 4-dimensional torus. (classical theory), hep-th/9807108.

[8] J. Ambjørn, Y.M. Makeenko, J. Nishimura and R.J. Szabo, Lattice gauge fields and discrete noncommutative Yang-Mills theory, JHEP 05 (2000) 023 [hep-th/0004147].

[9] P.M. Ho, Twisted bundle on quantum torus and BPS states in matrix theory, Phys. Lett. B 434 (1998) 41 [hep-th/9803166].

[10] D. Brace, B. Morariu and B. Zumino, Dualities of the matrix model from T-duality of the type-II string, Nucl. Phys. B 545 (1999) 192 [hep-th/9810099].

[11] S.B. Bradlow, Vortices in holomorphic line bundles over closed Kähler manifolds, Commun. Math. Phys. 135 (1990) 1.

[12] M. Chaichian, P. Prešnajder, M.M. Sheikh-Jabbari and A. Tureanu, Noncommutative gauge field theories: a no-go theorem, Phys. Lett. B 526 (2002) 132 [hep-th/0107037]. 